\title{
STUDY OF THYROID ASSOCIATED OPHTHALMOPATHY AND GLAUCOMA
}

\section{Rishendra singh Sisodiya ${ }^{1}$, Rajendra kumar samar ${ }^{* 2}$.}

${ }^{1}$ Assistant professor, Department of Ophthalmology, Pacific Institute of Medical sciences, Udaipur, Rajasthan, India.

${ }^{* 2}$ Assistant professor, Department of General Medicine, Pacific Institute of Medical sciences, Udaipur, Rajasthan, India.

\section{ABSTRACT}

Background: Glaucoma is an important cause of blindness world-wide, as a rise in intraocular pressure is associated with optic disc damage. However, the impact of this hypothyroidism on IOP in the setting of orbitopathy has not been clearly established.

Objective: To study the prevalence of glaucoma in patients with thyroid associated ophthalmopathy (TAO).

Methods: This cross sectional study includes total 86 patients with thyroid associated ophthalmopathy (TAO) who visited ophthalmology and medicine OPD of our Institute. All patients underwent complete ophthalmic examination including BCVA, Slit lamp examination, Fundus examination by $+78 \mathrm{D}$ lens, intraocular pressure measurement by Goldman Applanation Tonometer (GAT), visual fields (SITA standard 30-2, Humphrey, USA and pachymetry (Sonomed, USA). Exophthalmomery was done using Luedde's Exophthalmometer.

Results: A total of 86 consecutive patients of TAO were included in the study. There were 45 males and 41 females in our study. The mean age of the patients was 39.5 years (Range: $23-57.5$ years). All patients had some degree of proptosis along with congestive features of active TAO. In our study 52 out of 172 eyes had IOP $>21$ $\mathrm{mm} \mathrm{Hg}$. 21 eyes had optic nerve changes and visual field changes to be labeled as glaucomatous. 31 eyes had only high IOP without corresponding optic nerve or visual field changes.

Conclusions: Patients with thyroid associated ophthalmology (TAO) have high prevalence of primary open angle glaucoma, secondary glaucoma and ocular hypertension.

KEY WORDS: Glaucoma, Grave's disease, Thyroid associated ophthalmopathy.

Address for correspondence: Dr Rajendra kumar samar, 12, Shivpark, Durga Nursery road, Udaipur, Rajasthan-313001, India. E-Mail: rajendrasamar11111@gmail.com

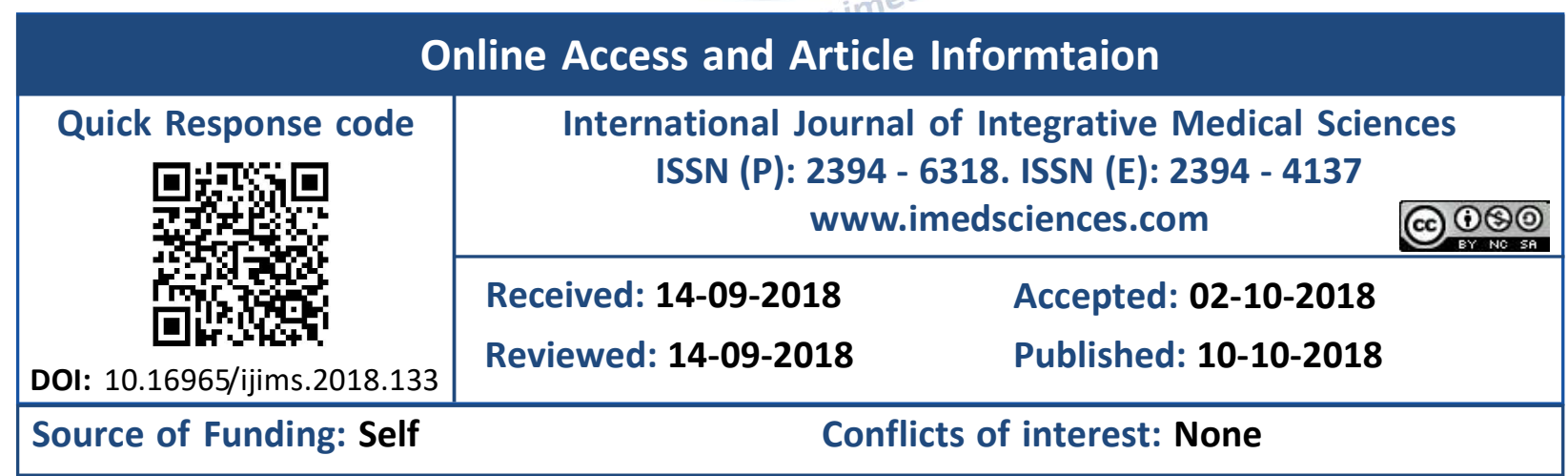

\section{BACKGROUND}

Glaucoma is an important cause of blindness world-wide, as a rise in intraocular pressure is associated with optic disc damage. Recently, a large study had indicated an association between hypothyroidism and glaucoma [1]. Hypothyroidism is also a common disease [2]. This article aims to put the issue of hypothyroidism and its link to glaucoma in perspective for the clinician treating this illness.

Hormones might affect the development of glaucoma and IOP through various mechanisms, and some studies have reported that thyroid hormone affects IOP. Thyroid hormones play a key role in metabolism and homeostasis [3]. Thyroid-associated ophthalmopathy has been described clinically; however, the relationship between thyroid function and glaucoma has 
remained controversial [4]. Some studies have reported a significant relationship, while others have not. Forte et al. [5], suggested that ocular hypertension (OHT) patients with Graves' orbitopathy showed diffuse abnormalities of the VF and RNFL thinning.

Thyroid hormone affects the maintenance of homeostasis in the body and regulates the basal body metabolism [6]. Many studies have tried to determine the association between hypothyroidism and glaucoma. In our study, we did not find that thyroid dysfunction was an independent risk factor of glaucoma development. On univariate analysis, the presence of glaucomatous optic neuropathy was significantly influenced by the level of T3, but the association did not remain statistically significant on multivariate regression. Disc haemorrhage was not associated with thyroid dysfunction as well.

\section{SUBJECTS AND METHODS}

This Cross sectional study was conducted at department of ophthalmology in association with medicine department of Pacific Institute of Medical sciences, Udaipur, Rajasthan ,India from January to June 2017.

In a cross sectional study, patients with thyroid associated ophthalmopathy (TAO)who visited eye OPD and medicine OPD were included in the study.

The diagnosis of TAO was based on clinical symptoms, signs, proptosis, eyelid retraction, ocular inflammation, the presence of goiter, elevated levels of serum T3 and T4, and other supporting features. All patients underwent complete ophthalmic examination including BCVA, Slit lamp examination, Fundus examination by +78 D lens, intraocular pressure measurement by Goldman Applanation Tonometer (GAT), visual fields (SITA standard 30-2, Humphrey, USA and pachymetry (Sonomed, USA). Exophthalmometry was done using Luedde's Exophthalmometer. The data thus obtained was tabulated and analysed using SPSS software.

Glaucoma was defined as IOP greater than 21 with glaucomatous changes of optic nerve and/ or glaucomatous visual fields defects. Ocular hypertension was defined as IOP greater than $21 \mathrm{~mm} \mathrm{Hg}$ without optic nerve changes and normal visual fields. Proptosis was defined as measurement of Luedde's exophthalmeter $>20 \mathrm{~mm}$ from lateral orbital rim.

A non contrast Computed tomography of orbits was done for evaluation of proptosis, extraocular muscle enlargement and globe compression whenever indicated. MRI orbit was done in few cases where optic nerve compression was suspected.

\section{RESULTS}

A total of 86 consecutive patients of TAO were included in the study. There were 45 males and 41 females in our study. The mean age of the patients was 39.5 years (range: 23-57.5 years). All patients had some congestive features of active TAO and varying degree of proptosis. (Table 1,2)

In our study 52 out of 172 (30.23\%) eyes had IOP > $21 \mathrm{~mm} \mathrm{Hg.} 21$ eyes (12.20\%) had optic nerve changes and visual field changes to be labeled as glaucomatous. 31 (18.02\%) eyes had only high IOP without corresponding optic nerve or visual field changes. (Table 3 )

Table 1: Distribution of participant based on sex.

\begin{tabular}{|c|c|c|}
\hline $\begin{array}{c}\text { Total no of } \\
\text { participants (n) }\end{array}$ & Male & Female \\
\hline 86 & 45 & 41 \\
\hline
\end{tabular}

Table 2: Age wise Distribution of participants.

\begin{tabular}{|c|c|c|}
\hline $\begin{array}{c}\text { Total no of } \\
\text { participants }(\mathrm{n})\end{array}$ & $\begin{array}{c}\text { Age Group } \\
(\mathrm{yr})\end{array}$ & $\begin{array}{c}\text { Mean Age } \\
(\mathrm{yr})\end{array}$ \\
\hline 86 & $23-57.5$ & 39.5 \\
\hline
\end{tabular}

Table 3: Ophthalological finding of participants

\begin{tabular}{|c|c|c|}
\hline parameter & Number & Percentage (\%) \\
\hline Total no of examined eyes & 172 & - \\
\hline IOP > 21 mmhg & 52 & 30.23 \\
\hline Glaucoma & 21 & 12.2 \\
\hline Ocular hypertension & 31 & 18.02 \\
\hline
\end{tabular}

Graph 1: Graphical presentation of ophthalmological finding.

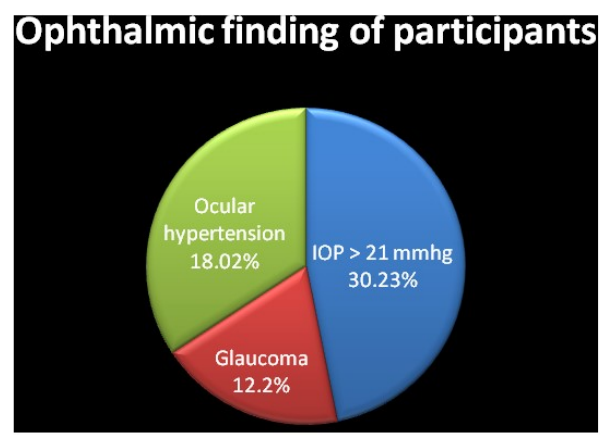




\section{DISCUSSION}

Thyroid over or under activity can be associated with problems in and around the eyes. When it occurs, it is known as "thyroid-associated ophthalmopathy. The socket in which the eyes are located can be affected by inflammation; fluid and white blood cells can collect in the tissues behind and alongside the eyes causing swelling. This swelling pushes the eyes forwards so that they bulge, the blood vessels around the eyes open wide making them look red and angry and the eyelids pull back. All this makes it very hard to close the eyes properly. The front windows of the eyes (the corneas) can't be protected adequately and roughness or even ulcers can form. These can become infected, and may heal leaving scars.

Physiologically, TSH level and T3 and T4 levels are adjusted in relation to each other [3]. In the hyperthyroid state, blood tests typically show low thyroid-stimulating hormone (TSH) and elevated triiodothyronine (T3) and thyroxine (T4) levels. Otherwise, an elevated TSH indicates that the thyroid gland is not producing enough thyroid hormone (hypothyroid state). In our study, the IOP in the first quartile of free T4 and T3 were higher than those in the second or other quartiles. These results were statistically significant, but the changes were clinically insignificant. These results can be considered the several reasons that might appear. First, this study included a very large number of subjects and the results for each group might be distributed evenly. Due to very large number of the enrolled subjects in the study, statistically significant result was showed. However, the difference was very small and might not be noticeable. Second, in normal population, the structural abnormalities of the extraocular muscles or intraorbital tissues that could affect the IOP were less likely to appear. Therefore, the difference of IOP between groups was less likely to emerge.

Considering previous reports about an association between IOP and thyroid function, there was a possible theoretical relationship between thyroid function and glaucoma. Hyperthyroidism might induce IOP increase through increased intraorbital pressure or contraction of enlarged extraocular muscles $[7,8]$.

The main mechanism could be elevation of episcleral venous pressure secondary to increasing of intraorbital content and pressure. The increased IOP in hypothyroidism could be due to the excessive accumulation of mucopolysaccharides in the trabecular meshwork $[9,10]$. Smith et al. [9] reported that the prevalence of hypothyroidism in the primary open-angle glaucoma group was higher than those in the control subjects in their case- control study. They proposed that, in the untreated hypothyroid state, hyaluronic acid accumulates excessively in the trabecular meshwork and/or aqueous, causing an obstruction to facility of outflow. This phenomenon was thought to be due to decreased enzyme activity in the hypothyroid state and decreased degradation of hyaluronic acid. After treatment of hypothyroidism, the outflow of aqueous humor was recovered. Some studies have reported that treating hypothyroidism decreases IOP through increasing aqueous outflow. Centanni et al. ${ }^{[1]]}$ reported that the IOP of subjects who showed a significantly higher IOP than the control group of subclinical hypothyroidism were decreased approximately by $3 \mathrm{mmHg}$ after treatment of hypothyroidism. Bahceci et al. [12] reported that hypothyroidism reversibly induced increasing IOP. On the other hand, Cheng and Perkins [13] did not find a statistically significant difference in the distribution of IOP between hypothyroidism and normal control groups. However, their study included thyroid hormone-treated patients, which could have affected the results due to the reversible effects of the treatment. McLenachan and Davies [14] reported that high IOP was associated with hypothyroidism due to changes in the quantity and quality of mucopolysaccharides in the trabecular meshwork.

\section{CONCLUSION}

From our study it will be conclude that the prevalence of Primary open angle glaucoma, secondary glaucoma and ocular hypertension is much higher among patients with Thyroid associated ophthalmopathy. Thus all patients with TAO must undergo IOP measurement and may be treated as required. 


\section{REFERENCES}

[1]. CrossJM, Girkin CA, Owsley C, McGwin G Jr. The association between thyroid problems and glaucoma. Br J Ophthalmol 2008;92:1503 5.

[2]. Unnikrishnan AG, Kalra S, Sahay RK, Bantwal G, John $M$, Tewari N. Prevalence of hypothyroidism in adults: An epidemiological study in eight cities of India. Indian J Endocrinol Metab 2013;17:647 52.

[3]. Leske M. C., Heijl A., Hussein M., et al. Factors for glaucoma progression and the effect of treatment: the early manifest glaucoma trial. Archives of Ophthalmology. 2003;121(1):48-56.

[4]. Nouri-Mahdavi K., Hoffman D., Coleman A. L., et al. Predictive factors for glaucomatous visual field progression in the Advanced Glaucoma Intervention Study. Ophthalmology. 2004;111(9):16271635.

[5]. Forte R., Bonavolonta P., Vassallo P. Evaluation of retinal nerve fiber layer with optic nerve tracking optical coherence tomography in thyroid-associated orbitopathy. Ophthalmologica. 2010;224(2): 116-121

[6]. Nouri-Mahdavi K., Hoffman D., Coleman A. L., et al. Predictive factors for glaucomatous visual field progression in the Advanced Glaucoma Intervention Study. Ophthalmology. 2004;111(9):1627-1635.

[7]. Ohtsuka K., Nakamura Y. Open-angle glaucoma associated with Graves disease. American Journal of Ophthalmology. 2000;129(5):613-617.

[8]. Lee H. B., Rodgers I. R., Woog J. J. Evaluation and management of Graves orbitopathy. Otolaryngologic Clinics of North America. 2006;39(5):923942. doi: 10.1016/j.otc.2006.07.008.
[9]. Smith K. D., Arthurs B. P., Saheb N. An association between hypothyroidism and primary open-angle glaucoma. Ophthalmology. 1993;100(10):15801584.

[10]. Kalmann R, Mourits MP. Prevalence and management of elevated intraocular pressure in patients with Graves' orbitopathy. Br J Ophthalmol 1998;82:754 -757.

[11]. Centanni M., Cesareo R., Verallo O., et al. Reversible increase of intraocular pressure in subclinical hypothyroid patients. European Journal of Endocrinology. 1997;136(6):595-598.

[12]. Bahceci U. A., Ozdek S., Pehlivanli Z., Yetkin I., Onol $M$. Changes in intraocular pressure and corneal and retinal nerve fiber layer thicknesses in hypothyroidism. European Journal of Ophthalmology. 2005;15(5):556-561.

[13]. Cheng H., Perkins E. S. Thyroid disease and glaucoma. The British Journal of Ophthalmology. 1967;51(8):547-553.

[14]. McLenachan J., Davies D. M. Glaucoma and the thyroid. The British Journal of Ophthalmology. 1965;49(8):441-444. nuw imeds

How to cite this article: Rishendra singh Sisodiya, Rajendra kumar samar. STUDY OF THYROID ASSOCIATED OPHTHALMOPATHY AND GLAUCOMA. Int J Intg Med Sci 2018;5(8):725-728. DOI: 10.16965/ ijims.2018.133 